\title{
Tunneling Images of Gallium on a Silicon Surface: Reconstructions, Superlattices, and Incommensuration
}

\section{Citation}

Chen, D. M., J. A. Golovchenko, P. Bedrossian, and K. Mortensen. 1988. Tunneling Images of Gallium on a Silicon Surface: Reconstructions, Superlattices, and Incommensuration. Physical Review Letters 61, no. 25: 2867-2874. doi:10.1103/physrevlett.61.2867.

\section{Published Version}

doi:10.1103/PhysRevLett.61.2867

\section{Permanent link}

http://nrs.harvard.edu/urn-3:HUL.InstRepos:29407037

\section{Terms of Use}

This article was downloaded from Harvard University's DASH repository, and is made available under the terms and conditions applicable to Other Posted Material, as set forth at http:// nrs.harvard.edu/urn-3:HUL.InstRepos:dash.current.terms-of-use\#LAA

\section{Share Your Story}

The Harvard community has made this article openly available.

Please share how this access benefits you. Submit a story.

\section{Accessibility}




\title{
Tunneling Images of Gallium on a Silicon Surface: Reconstructions, Superlattices, and Incommensuration
}

\author{
D. M. Chen, ${ }^{(a)}$ J. A. Golovchenko, P. Bedrossian, and K. Mortensen \\ Physics Department and Division of Applied Science, Harvard University, Cambridge Massachusetts 02138, \\ and Rowland Institute for Science, Cambridge Massachusetts 02142 \\ (Received 19 September 1988)
}

\begin{abstract}
Real-space images of an incommensurate superlattice on a monolayer-gallium-covered silicon (111) surface have been obtained with the tunneling microscope. Large, internally ordered supercells which in turn form a lattice with discrete boundaries are observed. A graphitelike silicon-gallium top layer is suggested that is weakly bonded to the lattice below and stabilized by a periodic array of misfit dislocations. This model unifies reflection high-energy electron diffraction, $x$-ray standing-wave, and tunneling observations.
\end{abstract}

PACS numbers: $68.35 . \mathrm{Rh}, 61.16 . \mathrm{Di}$

It has become clear in recent years that new phenomena and forms of matter appear to reside at surfaces and interface regions of solids. This is a challenging area of current research that promises to provide a greater understanding of electronic devices as well as heteroepitaxial growth processes. Here we report a structural study of monolayer quantities of gallium atoms deposited on an atomically clean (111) surface of a silicon crystal. Using the tunneling microscope, we show what we believe are the first atomic scale observations of an incommensurate superlattice on this system. Such a structure was first suggested on the basis of a wide variety of new diffraction spots observed in reflection high-energy electron diffraction studies by Otsuka and Ichikawa. ${ }^{1}$ The existence of incommensurate structures and the driving forces that create and stabilize them have recently attracted much attention. ${ }^{2,3}$

The experiments were performed in an ultrahigh vacuum chamber where both a low-energy electron diffraction (LEED) apparatus and a tunneling microscope reside. A number of scanning-tunneling-microscopy studies of metal-covered silicon surfaces have been reported recently. ${ }^{4-8}$ In our case the silicon (111) samples (arsenic doped to $0.002 \Omega-\mathrm{cm}$ ) were sputter cleaned in a $1-\mathrm{keV}$ neon beam and annealed at $2 \times 10^{-10}$ Torr. The standard $7 \times 7$ pattern was observed with the LEED apparatus as well as the tunneling microscope. The preparation results in large, reproducible, reconstructed, surface regions with atomic steps typically spaced at thousand-angstrom intervals.

Gallium atoms were than deposited on the surface by evaporation from an effusion cell at $700^{\circ} \mathrm{C}$ while the substrate was held at $\approx 300^{\circ} \mathrm{C}$. After the experiment coverages were evaluated by in situ Auger spectroscopy and by Rutherford backscattering. The average coverages and associated surface phases are in all cases consistent with those reported previously. ${ }^{1,9,10}$

As the surface coverage was increased to $\frac{1}{3}$ monolayer (ML) $\left(1 \mathrm{ML}=7.8 \times 10^{14}\right.$ gallium atoms $\left./ \mathrm{cm}^{2}\right)$ the diffraction pattern transformed to a $\sqrt{3} \times \sqrt{3} R 30^{\circ}$ pattern originally seen by Lander and Morrison. ${ }^{11}$ Increasing the coverage of gallium atoms up to $1 \mathrm{ML}$ and subsequent annealing at $300-500^{\circ} \mathrm{C}$ resulted in a diminution of the intensity of the $\sqrt{3} \times \sqrt{3} R 30^{\circ}$ diffraction spots with a corresponding formation of sets of hexagonal arrays of spots centered about the first-order diffraction spots of the underlying silicon lattice. We associate these spots with the $6.3 \times 6.3$ and other periodicities reported in Ref. 1.

Figure 1 displays a typical tunneling image obtained from a sample showing such a diffraction pattern with an average gallium coverage of $1 \mathrm{ML}$. The small thermal drift has not been corrected in this or subsequent figures.

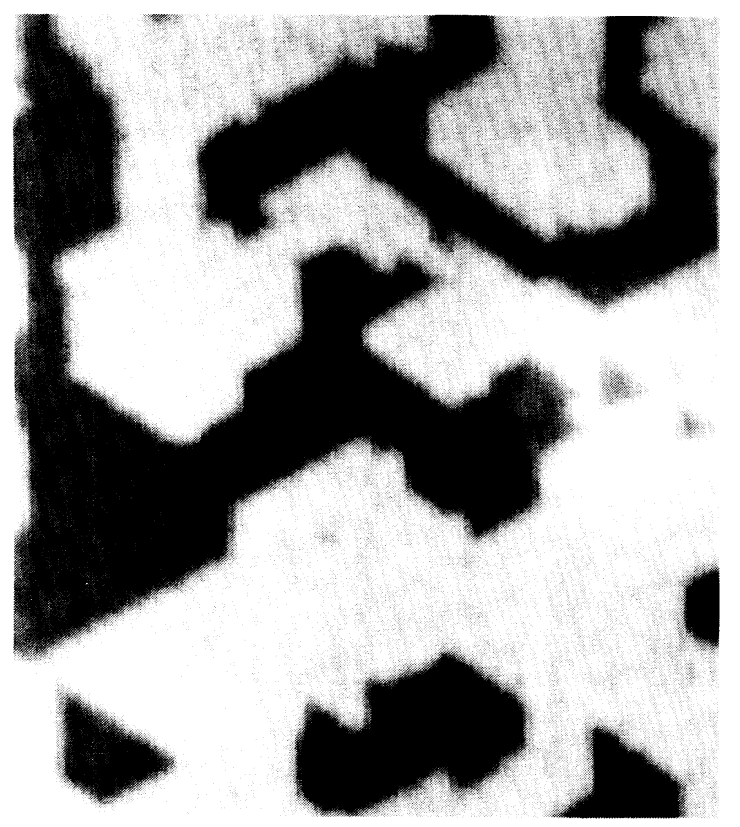

FIG. 1. $470 \times 400-\AA^{2}$ tunneling image of 1-ML Ga-covered $\mathrm{Si}(111)$, showing the overlayer superlattice of $24-\AA$ periodicity, with $(3.2 \pm 0.2)$ - $\AA$ step heights. The tip bias and tunneling current are $-3.32 \mathrm{~V}$ and $800 \mathrm{pA}$, respectively. 
Ordered layers of hexagonally close-packed aggregates, henceforth referred to as supercells, are evident. The superlattice unit cells are aligned with the threefold symmetry axes of the bulk, and their size is approximately $24 \AA$. We identify the superlattice periodicity with the $6.3 \times 6.3$ reflection high-energy electron diffraction observations in Ref. 1 as well as our own LEED observations on this sample. The step height between layers is $3.2 \pm 0.2 \AA$, which coincides with the bulk silicon (111) spacing. The shape of the individual supercells is well defined right up to the step edges, which appear remarkably straight and align with the symmetry directions. At the boundary between the supercells the tip drops down of order $0.75 \AA$. Often the superlattice also displays regions of weaker long-range periodicity together with
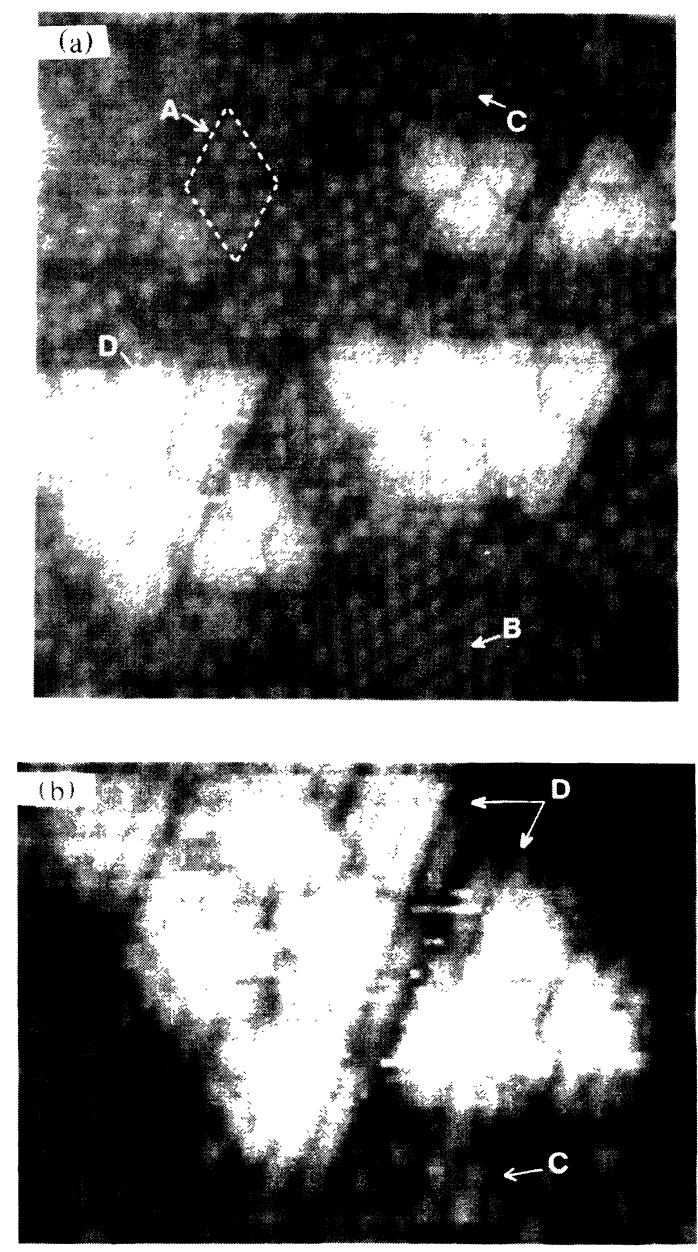

FIG. 2. (a) Tunneling image of a sample with $\frac{1}{2}-M L G a$ coverage, exhibiting $7 \times 7$ unit cells (A), regions of $\sqrt{3}$ $\times \sqrt{3} R 30^{\circ}$ reconstruction (B), nucleation sites (C), and islands of supercells (D). The scale is $180 \times 200 \AA^{2}$, while the tip bias and tunneling current were $-1.5 \mathrm{~V}$ and $1 \mathrm{nA}$. (b) Higherresolution scan, $100 \times 80 \AA^{2}$, showing constituent atoms of the supercells. The tip bias is $-1.75 \mathrm{~V}$. more irregular supercell shapes and sizes.

In order to explore the initial growth stages of the new superlattice, we prepared a sample with an average $\frac{1}{2}$ ML gallium coverage that yielded both $7 \times 7$ and $\sqrt{3} \times \sqrt{3} R 30^{\circ}$ LEED spots simultaneously. The coexistence of LEED patterns is due to local nonuniformity of coverage, as shown in Fig. 2(a), a representative tunneling image of this surface. In addition to a sea of somewhat disordered $7 \times 7$ unit cells (A), we observe first regions (B) of $\sqrt{3} \times \sqrt{3} R 30^{\circ}$ gallium adatoms occupying the $T_{4}$ site, confirming our previous report. ${ }^{8}$ Second, we find what appear to be gallium nucleation sites $(C)$ in the adatom layers of the $7 \times 7$ reconstruction, which are similar to those recently reported. ${ }^{6,7}$ Third, we observe islands of the new superlattice structure (D), above which the tunneling tip retracts $1.2 \AA$ relative to its position above the other adatoms on the surface. As evident from Fig. 2(b), a higher-resolution scan of this region
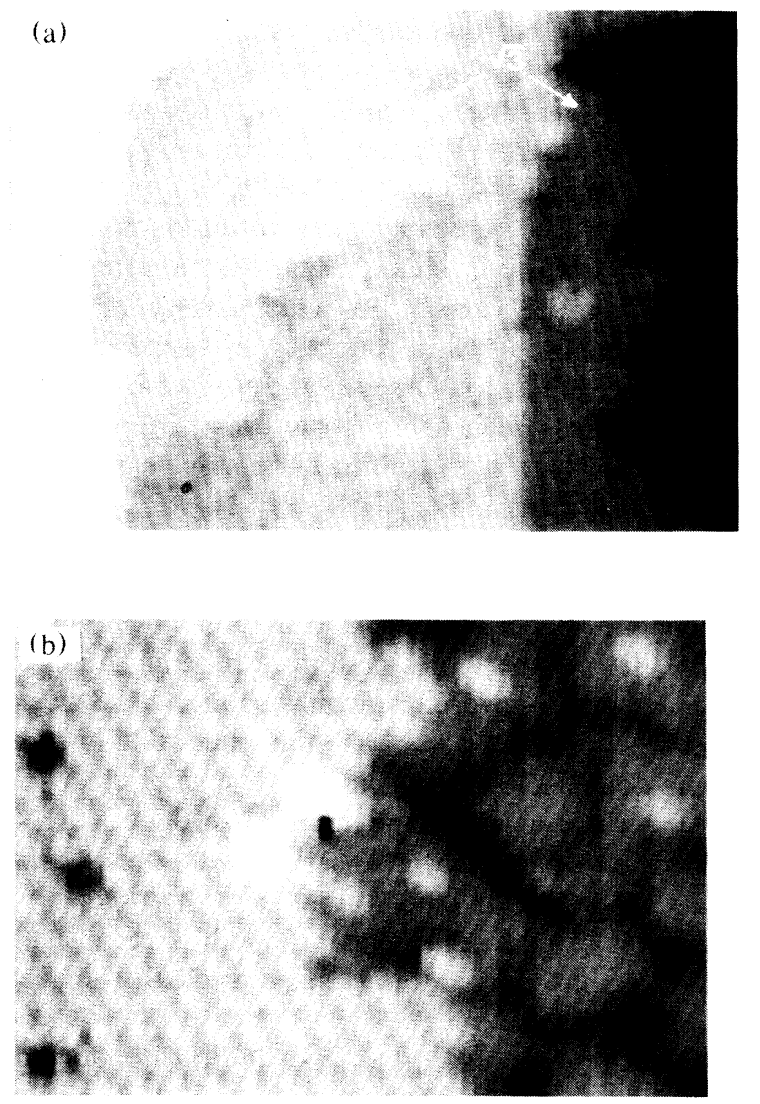

FIG. 3. Tunneling images obtained after flash heating a Ga-covered $\mathrm{Si}(111)$ surface of the type in Fig. 1. (a) 450 $\times 300-\AA^{2}$ scan, taken at a tip bias of $-2.5 \mathrm{~V}$ and a tunneling current of $1 \mathrm{nA}$, showing $\sqrt{3} \times \sqrt{3} R 30^{\circ}$ planes occurring at steps between superlattice terraces. (b) The left portion of this $120 \times 90-\AA^{2}$ scan shows a $\sqrt{3} \times \sqrt{3} R 30^{\circ}$ atomic plane $1.5 \AA$ above the superlattice layer exposed on the right-hand side. The tip bias is $-3.2 \mathrm{~V}$. 
showing individual atoms, the supercells are in turn composed of an oriented, hexagonal array of lattice spacing 4.1 $\AA$. High-resolution images of supercells in extended superlattices also show this internal structure.

Finally, Fig. 3 shows the result of a flash heating $\left(600^{\circ} \mathrm{C}, 10 \mathrm{~s}\right)$ of a surface initially covered completely by the superlattice. This treatment resulted in a reappearance of $\sqrt{3} \times \sqrt{3} R 30^{\circ}$ diffraction spots in addition to the superlattice spots. In addition to the features observed in Fig. 1, a new region, $1.1 \AA$ above the superlattice, on the right-hand side, now appears in tunneling images such as Fig. 3(a). A higher-resolution scan shown in Fig. 3(b) reveals that the dimensions and orientation of the unit cells in this region are identical to those of $\sqrt{3} \times \sqrt{3} R 30^{\circ}$ gallium on silicon, confirming the LEED observations. Furthermore, regions of this reconstruction were found only adjacent to edges of superlattice terraces, as is evident from Fig. 3(a).

Overall, the tunneling images of the new superlattice material show a startling diversity of structure. The detailed nature of the observed supercells and boundaries that compose the incommensurate superlattice must derive from the competition between adsorbate-adsorbate and adsorbate-substrate interactions. The $x$-ray standing-wave results of Ref. 9 were interpreted as arising from a $1 \times 1$ double-layer structure with gallium atoms at transverse outer substitutional sites, but only $0.29 \AA$ above the silicon layer which is not observed. Our tunneling images, however, show a larger transverse lattice constant of $4.1 \AA$ rather than the $3.8 \AA$ of the silicon bulk.

A natural explanation for both observations incorporates a compression of the outer double layer by $\geq 0.5 \AA$ and a significant lateral expansion in order to relieve the resulting compressive stress. This is presumably connected with a greater weakening of the bonding between this top layer and the bulk then previously anticipated. ${ }^{9}$ In fact, preliminary calculations ${ }^{12}$ predict extremely large compressive stress for the $1 \times 1$ double-layer model proposed in Ref. 9, suggesting that a large transverse relaxation of the surface is very likely. We thus think of the supercells as consisting of two nearly coplanar interpenetrating hexagonal silicon and gallium lattices that create a graphitelike surface with a lattice constant as observed in the images. Finally, we note that an inert graphitelike structure at the surface may well explain the inability of gallium to wet a silicon surface past $1 \mathrm{ML} .^{13}$

According to this picture the steps observed in Fig. 1 would naturally have the silicon (111) periodicity for a step height, as measured. A natural explanation for the high step density apparent in Fig. 1 but absent from the virgin substrate also emerges from this model, for silicon atoms that are freed up in the formation of the lower terrace can contribute to the formation of an upper terrace. Upon flash heating, desorption of gallium atoms initiated near the steps precipitates the reversal of the preceding process. ${ }^{13}$ Coverage of the resulting fresh silicon double layer by remaining gallium atoms would then yield the $\sqrt{3} \times \sqrt{3} R 30^{\circ}$ pattern observed in Fig. 3 .

The incommensurate relationship and the interaction between the proposed graphitelike structure and the silicon lattice below will provide a driving force to create new periodicities in the system. The observed boundaries of the incommensurate superlattice cells every $24 \AA$ then arise from surface dislocations (rather than a simple moiré effect which, based on a 4.1 - $\AA$ surface lattice constant, would give rise to a $40-\AA$ superlattice periodicity instead). The interaction responsible for the creation of surface dislocations may be such a violent function of lateral overlayer displacement as to preclude a simple microscopic explanation for the observed superlattice periodicities and boundary structure. At least we have none at this time.

In addition to the $6.3 \times 6.3$ superlattice, we also note our observation of the diffraction patterns previously assigned $^{1}$ as $11 \times 11$ and $6.3 \sqrt{3} \times 6.3 \sqrt{3} R 30^{\circ}$ for various surface preparations below $1 \mathrm{ML}$. The $11 \times 11$ spots may be caused by the $4.1-\AA$ internal periodicity of the supercells (with multiple scattering taken into account in the LEED interpretation). While we have not seen obvious manifestations of the other symmetry in our tunneling images, we note that the irregular supercells mentioned earlier are candidates for new extended periodicities as are subsurface silicon atom reconstructions that we do not observe with the microscope. This indicates that this system can take up rather complicated phases for which our model may supply only a partial explanation. We have attempted to obtain further information on the structure of this surface from local tunneling $I-V$ spectra, but at bias values below $1.5 \mathrm{~V}$, tunneling stability decreases drastically and reproducible results in that interesting energy region are not yet available. At any rate, we suggest that further study of this system by other microscopic methods, for example extended x-rayabsorption fine-structure spectroscopy, core-level spectroscopy, photoemission, etc., may further illuminate this interesting new interfacial region.

In conclusion, we have observed spatial images of new incommensurate superlattice structures on a galliumcovered silicon (111) lattice. These appear to be caused by the interaction of a graphitelike layer of a new phase of $\mathrm{Si}-\mathrm{Ga}$ with the substrate below. Periodic arrays of presumed misfit dislocations decorate the boundary between superlattice cells whose detailed characterization and understanding challenge present understanding of surface phenomena. Our observations also suggest that the tunneling microscope is a powerful tool for learning how to produce, pin, or eliminate such dislocations in epitaxial material by proper interface phase preparation methods.

We would like to thank J. R. Patel, John Chervinsky, David Vanderbilt, and members of the Rowland Institute 
for valuable cooperation and discussion. This work was supported by ONR Grant No. N00014-87-K-0511 and Harvard Materials Research Laboratory Grant No. DMR-86-14003. We acknowledge the financial support of P.B. by the NSF, and of K.M. by NATO.

(a) Permanent address: Department of Physics, University of Western Ontario, London, Canada N6A 3K7.

${ }^{1}$ M. Otsuka and T. Ichikawa, Jpn. J. Appl. Phys. 24, 1103 (1985).

${ }^{2}$ Hawoong Hong, C. J. Peters, A. Mak, R. J. Birgeneau, P. M. Horn, and H. Suematsu, Phys. Rev. B 36, 7311 (1987); also P. W. Stephens et al., Phys. Rev. B 29, 3512 (1984).

${ }^{3}$ S. N. Coopersmith, Daniel S. Fisher, B. I. Halperin, P. A. Lee, and W. F. Brinkman, Phys. Rev. B 25, 349 (1982).

${ }^{4}$ R. J. Wilson and S. Chiang, Phys. Rev. Lett. 59, 2329 (1987).

${ }^{5}$ J. Nogami, Sang-il Park, and C. F. Quate, Phys. Rev. B 36, 6221 (1987).
${ }^{6}$ U. K. Koehler, J. E. Demuth, and R. J. Hamers, Phys. Rev. Lett. 60, 2499 (1988).

${ }^{7}$ St. Tosch and H. Neddermeyer, Phys. Rev. Lett. 61, 349 (1988).

${ }^{8}$ J. E. Zegenhagen, J. R. Patel, Paul Freeland, D. M. Chen, J. A. Golovchenko, P. Bedrossian, and J. E. Northrup, Phys. Rev. B (to be published).

${ }^{9}$ J. Zegenhagen, M. S. Hybertsen, P. E. Freeland, and J. R. Patel, Phys. Rev. B 38, 7885 (1988).

${ }^{10}$ Coverage measurements contain contributions from gallium droplets, as seen in our scanning-tunneling-microscopy experiment. See also M. Zinke-Allmang and L. C. Feldman, Surf. Sci. 191, L749 (1987); also M. Zinke-Allmang, L. C. Feldman, S. Nakahara, and J. R. Patel, in Epitaxy of Semiconductor Layered Structures, edited by R. T. Tung, R. Dawson, and R. L. Gunshor, MRS Symposium Proceedings Vol. 102 (Materials Research Society, Pittsburgh, PA, 1988), p. 431.

11 J. J. Lander and J. Morrison, Surf. Sci. 2, 553 (1964).

${ }^{12} \mathrm{D}$. Vanderbilt, private communication.

${ }^{13}$ See references cited in Ref. 10. 


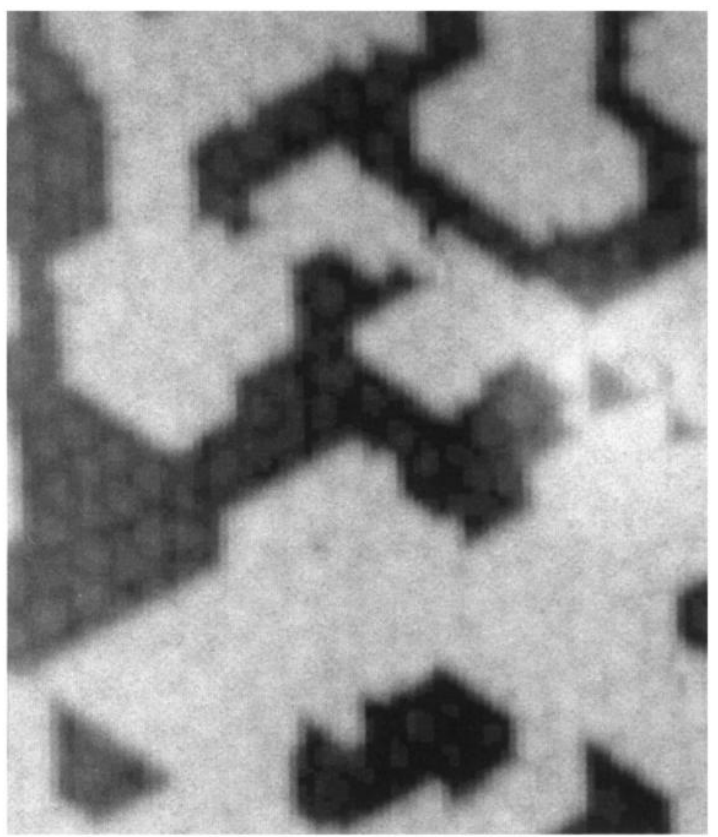

FIG. 1. $470 \times 400-\AA^{2}$ tunneling image of 1-ML Ga-covered $\mathrm{Si}(111)$, showing the overlayer superlattice of $24-\AA \AA$ periodicity, with $(3.2 \pm 0.2)-\AA$ step heights. The tip bias and tunneling current are $-3.32 \mathrm{~V}$ and $800 \mathrm{pA}$, respectively. 

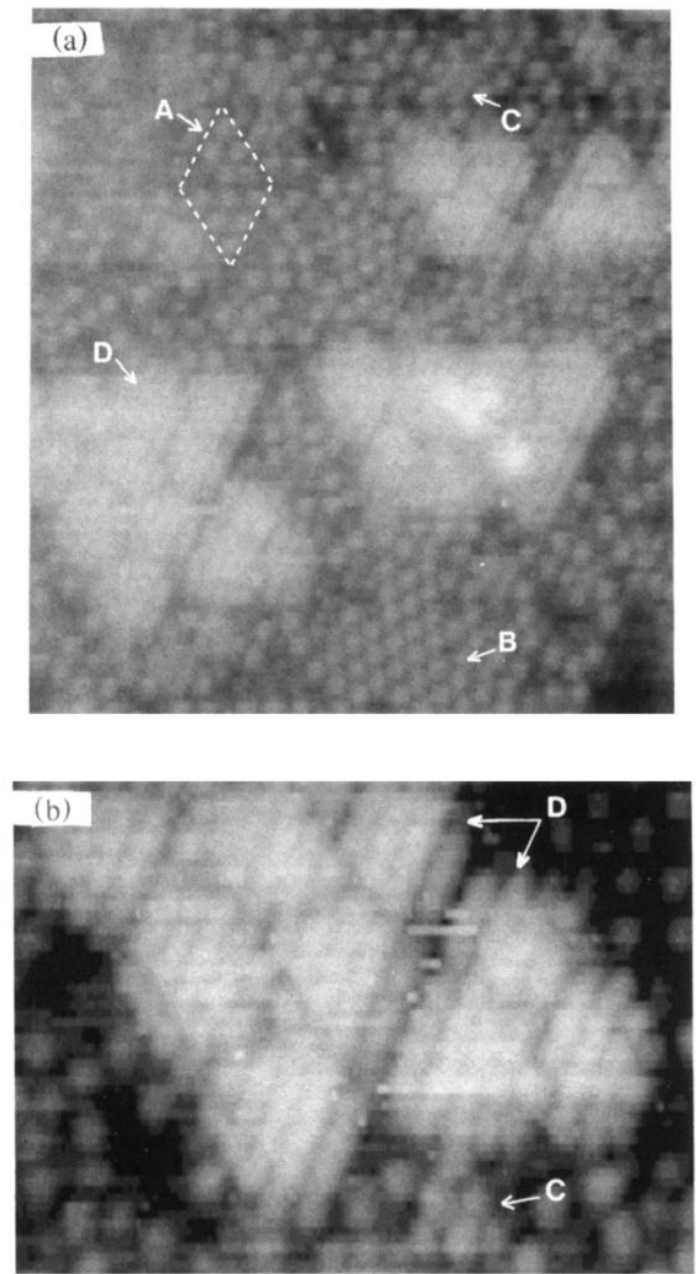

FIG. 2. (a) Tunneling image of a sample with $\frac{1}{2}-\mathrm{ML} \mathrm{Ga}$ coverage, exhibiting $7 \times 7$ unit cells (A), regions of $\sqrt{3}$ $\times \sqrt{3} R 30^{\circ}$ reconstruction (B), nucleation sites (C), and islands of supercells (D). The scale is $180 \times 200 \AA^{2}$, while the tip bias and tunneling current were $-1.5 \mathrm{~V}$ and $1 \mathrm{nA}$. (b) Higherresolution scan, $100 \times 80 \AA^{2}$, showing constituent atoms of the supercells. The tip bias is $-1.75 \mathrm{~V}$. 

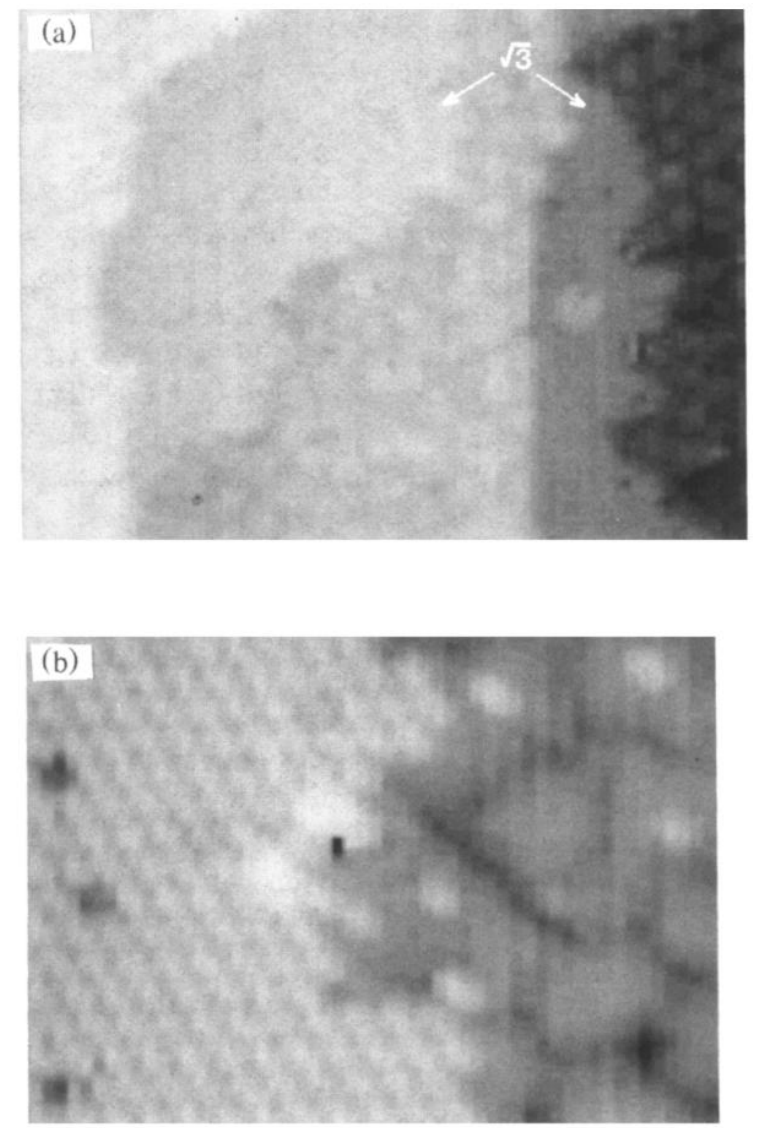

FIG. 3. Tunneling images obtained after flash heating a Ga-covered $\mathrm{Si}(111)$ surface of the type in Fig. 1. (a) 450 $\times 300-\AA^{2}$ scan, taken at a tip bias of $-2.5 \mathrm{~V}$ and a tunneling current of $1 \mathrm{nA}$, showing $\sqrt{3} \times \sqrt{3} R 30^{\circ}$ planes occurring at steps between superlattice terraces. (b) The left portion of this $120 \times 90-\AA^{2}$ scan shows a $\sqrt{3} \times \sqrt{3} R 30^{\circ}$ atomic plane $1.5 \AA$ above the superlattice layer exposed on the right-hand side. The tip bias is $-3.2 \mathrm{~V}$. 